\title{
It Takes More than Nudging Patients to Reduce Medication Non-adherence
}

\section{James X Zhang*}

Department of Medicine, The University of Chicago, Chicago, USA

\section{Editorial}

Medication non-adherence is a serious and elusive problem in the U.S. healthcare system [1]. The literature shows significant medication non-adherence among a wide range of patient populations, with an average non-adherence rate nationally of $24.8 \%$ and rates in some sub-populations as high as 95\% [2,3]. Despite efforts to increase health insurance coverage through the Medicare Part D outpatient prescription program and Medicaid expansion, as well as numerous efforts to improve patient-physician communication and patient education, medication non-adherence proves to be a persistent challenge as successful intervention are generally substantially complex and costly [4-6].

A recent study published in JAMA Internal Medicine represents another effort to address medication non-adherence [7]. This study, the REMIND study, using an elegant 4-arm, block-randomized clinical trial design, enrolled 53,480 patients who received prescription drugs through CVS Caremark, a pharmacy benefit manager, across the United States. Patients in the treatment group were randomly assigned to receive a pill bottle strip with toggles, a digital timer cap, or a standard pillbox. The control group received neither notification nor a device. Data were collected from February 12, 2013 through March 21, 2015, and data analyses were on the intention-to-treat population [7]. The results showed that there was no statistically significant difference in the odds of optimal adherence between the control and those who received any of the devices [7].

The REMIND study is by far the largest study to date that involved a rigorously executed clinical trial aimed at reducing medication nonadherence. There may be many reasons the study may not have shown any difference between the treatment groups and the control group. The authors suggested that adherence dynamics maybe a factor as patients' non-adherence behavior cannot be determined at one time point only; rather, it has to be analyzed and predicted longitudinally. The authors also reasoned that patients may not even be adherent to the devices they were given, which is consistent with the known behaviors in medication non-adherence.

There may be other factors to consider. For example, a recent study suggested that there are significant gender differences in behavior with non-adherence to medical care [8]. Since gender is a key determinant of many healthcare behaviors and outcomes, understanding variation in medication non-adherence by gender may provide opportunities to reduce disparities and improve outcomes [8]. The REMIND study did not report if women and men responded differently to the reminders. More importantly, gender-specific interventions may be more effective than generic approaches because they can generate differential responses that address gendered differences in biological and social needs.

Furthermore, the literature also suggests that many patients attribute medication non-adherence to cost [9]. If the root cause of non-adherence is an economic one, a single reminder system may not be sufficient or may not be addressing the key risk factor for non-adherence. Some of patients may be extremely sensitive to outof-pocket payments, given their socio-economic profile. Hence the REMIND system, which nudges the patients by helping them overcome forgetting or inertia, may need to be coupled with other interventions that give patients economic incentives or relief at the individual level in order to make it effective.

Overall, the factors that influence medication non-adherence are many and often difficult to measure and address. The REMIND study gives an excellent example of the fact that patients may need a multi-factorial intervention to move them through the barriers to medications use, which can be a mix of biological, social, economic, and psychological factors.

\section{References}

1. Gellad WF, Grenard J, McGlynn EA (2009) A review of barriers to medication adherence: a framework for driving policy options. RAND Corporation. Retracted from: http://www.rand.org/pubs/technical reports/TR765.html. Accessed January 31, 2018.

2. DiMatteo MR (2004) Variations in patients' adherence to medical recommendations: a quantitative review of 50 years of research. Med Care 42: $200-209$

3. Take As Directed: A Prescription Not Followed (2006) Research conducted by The Polling Company. National Community Pharmacists Association.

4. Madden JM, Graves AJ, Ross-Degnan D, Briesacher BA, Soumerai SB (2009) Cost-related medication nonadherence after implementation of Medicare Part D, 2006-2007. JAMA 302: 1755-1756

5. Tomaszewski M, White C, Patel P, Masca N, Damani R, et al. (2014) High rates of non-adherence to antihypertensive treatment revealed by high-performance liquid chromatography-tandem mass spectrometry (HP LC-MS/MS) urine analysis. Heart 100: 855-861.

6. Osterberg L, Blaschke T (2005) Adherence to medication. N Engl J Med 353 487-497.

7. Choudhry NK, Krumme AA, Ercole PM, Girdish C, Tong AY, et al. (2017) Effect of reminder devices on medication adherence: The REMIND randomized clinical trial. JAMA Intern Med 177: 624-631.

8. Zhang JX, Crowe JM, Meltzer DO (2017) The differential rates in cost-related non-adherence to medical care by gender in the US adult population. $\mathrm{J}$ Med Econ 20: 752-759

9. Aikens JE, Piette JD (2009) Diabetic patients' medication underuse, illness outcomes, and beliefs about antihyperglycemic and antihypertensive treatments. Diabetes Care 32: 19-24.
*Corresponding author: James X. Zhang, PhD, MS, Department of Medicine, 5841 S. Maryland Ave., MC 5000, Chicago, IL, 60637, USA, Tel: 1-7738341631; E-mail: xzhang1@medicine.bsd.uchicago.edu

Received March 09, 2018; Accepted March 13, 2018; Published March 16, 2018

Citation: Zhang JX (2018) It Takes More than Nudging Patients to Reduce Medication Non-adherence. J Gerontol Geriatr Res 7: e149. doi:10.4172/21677182.1000e149

Copyright: ( 2018 Zhang JX. This is an open-access article distributed under the terms of the Creative Commons Attribution License, which permits unrestricted use, distribution, and reproduction in any medium, provided the original author and source are credited. 